\title{
Short-term and long-term effects of a context-based vocabulary intervention for fifth-grade students with poor vocabulary knowledge
}

\section{ANNA STEENBERG GELLERT, SIGNE WISCHMANN AND ELISABETH ARNBAK}

The study investigated the effects of a 24 session context-based vocabulary intervention for fifth-graders with poor Danish vocabulary knowledge. The aim of the intervention was twofold: 1) to activate and strengthen students' knowledge of words within semantic fields and 2) to teach students how to derive the meaning of unfamiliar words from context during text reading. 111 students participated in the context-based intervention and were compared to 221 other students who either took part in an alternative vocabulary intervention or did not receive any intervention. The students were assessed at pretest, at posttest shortly after intervention, and at a follow-up assessment ten months after the intervention was completed. Results indicated large short-term effects of the context-based intervention on participants' reading and definition of taught words and a medium to large effect on their comprehension of texts containing such words. Notably, significant long-term effects were also found. However, similar to the results of previous studies, no transfer effects were found on participants' general vocabulary or general reading comprehension. Neither did the context-based intervention produce significant effects on a test of students' abilities to derive the meaning of unfamiliar words from context. Probably, such effects would require a more comprehensive training program.

KEYWORDS: word knowledge, reading comprehension, vocabulary intervention, context clues, training effects 